\title{
A sustainable route to inorganic porous hollow fibers with superior properties
}

\author{
Hussein Qasim Hussein ${ }^{\ddagger}$, Patrick de Wit ${ }^{\ddagger}$, Emiel J. Kappert, Nieck E. Benes*
}

Films in Fluids / Inorganic Membranes, Department of Science and Technology, MESA ${ }^{+}$ Institute for Nanotechnology, University of Twente, P.O. Box 217, 7500 AE Enschede, The Netherlands *n.e.benes@utwente.nl ${ }^{\ddagger}$ These authors contributed equally..

\section{Abstract}

This paper presents a method for the fabrication of inorganic porous hollow fibers, using ecologically benign feed materials instead of organic solvents and harmful additives. Our method is based on ionic cross-linking of an aqueous mixture of sodium alginate, inorganic particles, and a carbonate. The mixture is spun into an acidic coagulation bath, where the low $\mathrm{pH}$ triggers the dissociation of the carbonate into multivalent cations and carbon dioxide. The multivalent cations cross-link the alginate, thereby consolidating the 3D structure and arresting the inorganic particles. In a subsequent thermal treatment the polymer is removed and the particles are sintered together. Adequate gelation requires a sufficiently low $\mathrm{pH}$ of the acid bath and a sufficing buffering capacity of the acid. In addition, to facilitate thermal treatment, it appears to be crucial that the acid has a conjugated base with limited propensity for complexing cations. The environmentally-safe and sustainable lactic acid and acetic acid are shown to be convenient acids. The fibers prepared via our method have outstanding properties, such as high mechanical strength, homogeneous morphology, and sharp distribution of small pores. In addition, they are prepared using sustainable chemicals such as lactic acid and calcium carbonate.

Keywords: ionic cross-linking, alginate, porous inorganic hollow fiber, acid-carbonate reactions 


\section{Introduction}

Inorganic porous hollow fibers provide large surface-area-to-volume ratios, due to their small radial dimensions. As a result these fibers are applied in a vast array of applications, for example: photocatalysis, ${ }^{1,2}$ bio-reactor and sensors ${ }^{3,4}$, gas-liquid contactors, ${ }^{5-7}$ membranes for demanding separations ${ }^{8-10}$, and for combined chemical reaction and molecular separation in harsh environments. ${ }^{11-13}$ The small radial dimensions of the fibers are generally achieved by using the dry-wet spinning method, in which a mixture of a polymer, solvent and inorganic particles is spun into a coagulation bath. In the coagulation bath, non-solvent induced phase separation (NIPS) occurs and the inorganic particles are arrested in a polymer matrix. In a subsequent thermal treatment, the polymer is decomposed and the inorganic particles are sintered together. This method is well understood and described in detail for a variety of inorganic particles. ${ }^{14-16}$ The major drawback of the NIPS-method is the use of organic solvents. Typically, aprotic solvents such as dimethyl formamide (DMF), N-methyl-2-pyrrolidone (NMP) or dimethyl acetamide (DMAc) are used, because these dissolve a wide variety of polymers and the obtained spinning solutions coagulate readily upon contact with water.

We have recently demonstrated bio-ionic gelation as a novel solvent-free alternative approach for the fabrication of porous hollow fibers. ${ }^{17}$ The concept is based on the solidification of a spinning mixture by the ionic cross-linking of a sodium alginate when it is spun into an aqueous solution containing multivalent cations $\mathrm{M}^{n+}$, such as $\mathrm{Ca}^{2+}, \mathrm{Ba}^{2+}, \mathrm{Cu}^{2+}$, or $\mathrm{Fe}^{3+}$. The alginate is the sodium salt of the polysaccharide alginic acid that is produced by brown algae and bacteria and is a linear block copolymer consisting of 1,4-(ß-D)-mannuronic acid and 1,4-( $\alpha$-L)guluronic acid. Upon entering the solution, the multivalent cations diffuse into the spinning mixture and exchange with the sodium ions in the polymer, thereby forming a stable waterinsoluble three-dimensional gel network. ${ }^{18}$ This concept is depicted in Figure 1, top right. 
During a subsequent thermal treatment the biopolymer is removed and the inorganic particles are sintered together.

Introducing the multivalent ions into the alginate from a bath is known to have disadvantages. The gelation rate is hard to control and the concentration gradients of the multivalent ions inside and outside the alginate solution result in an inhomogeneous alginate gel. ${ }^{19-21}$ As a result of this, the mechanical integrity of the final fibers can be poor. ${ }^{17}$ In addition, in order to maintain stationary spinning conditions a regeneration of relatively large volumes of the $\mathrm{M}^{n+}$ solution is necessary.

In the present paper, we propose a new method for cross-linking fibers that overcomes these limitations, by providing the multivalent ions as an inactive, insoluble salt in the spinning mixture in the form of a carbonate. Carbonates have been used in the past for the production of homogenous microspheres, ${ }^{22-24}$ hydrogels ${ }^{25-27}$ and thin films. ${ }^{28-30}$ In our case, the mixture is spun into an acidic coagulation bath, where the low $\mathrm{pH}$ triggers the dissociation of the carbonate into $\mathrm{M}^{n+}$ and carbon dioxide. ${ }^{31}$ The multivalent ions are responsible for the ionic crosslinking of the biopolymer. The bottom right part of Figure 1 schematically depicts this concept. Gels prepared using the internal supply of multivalent ions are found to be more homogenous as compared to those obtained using the external supply. ${ }^{21}$ 

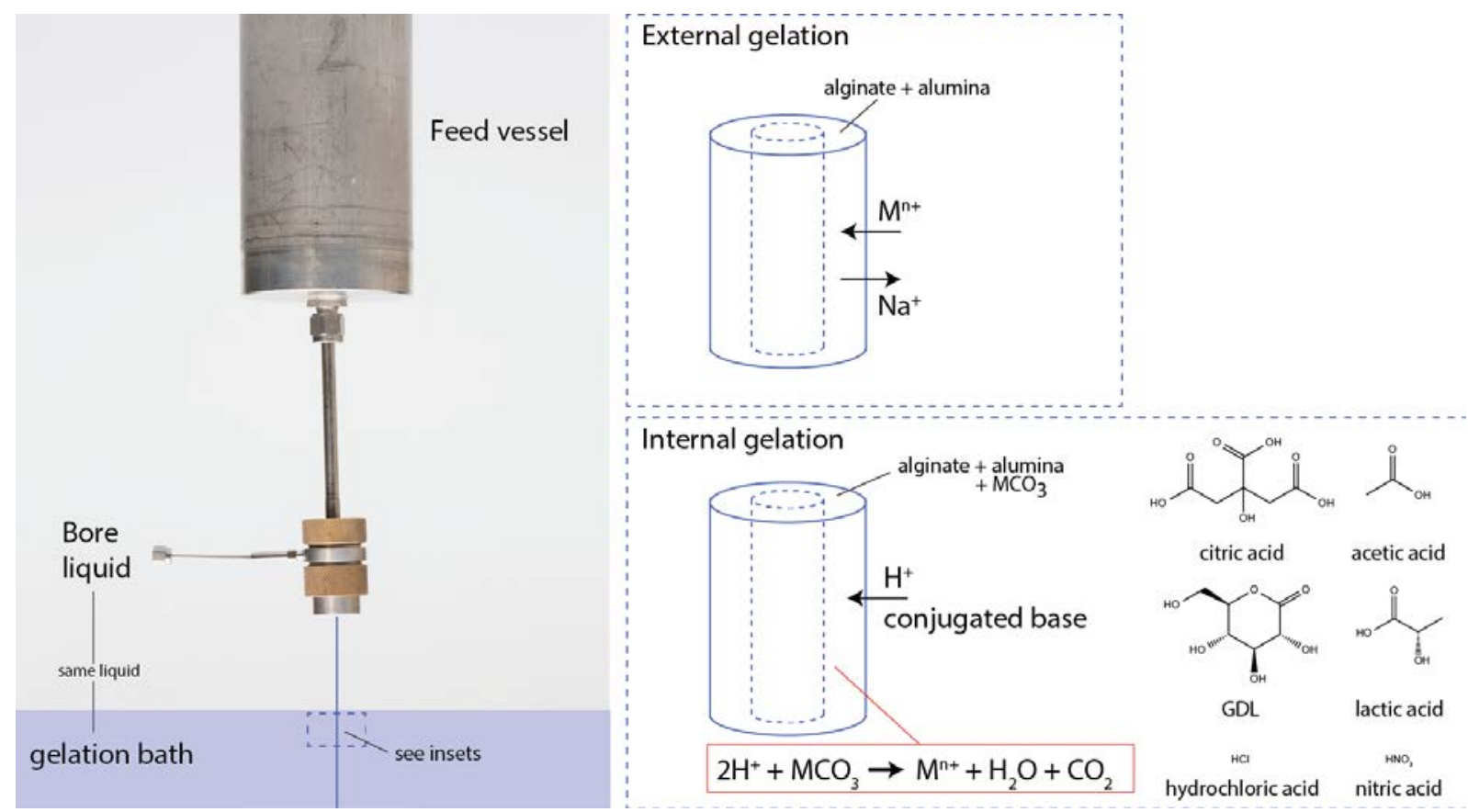

Figure 1. Left: set-up for dry-wet spinning, right: schematic representation of the concept of external (top right) and internal (bottom right) gelation. The spinning mixture is crosslinked with a solution of $\mathbf{M}^{\mathbf{n}+} \mathrm{Cl}_{2 n}$ in case of external gelation, whereas in the case of internal gelation the multivalent ion is present as $\mathrm{M}^{n+}\left(\mathrm{CO}_{3}\right)_{0.5 n}$ in the spinning mixture, which is in turn liberated upon contact with protons originating from the gelation bath.

To demonstrate the versatility of the proposed method, we compare different types of carbonates, and different types and concentrations of acids. Conforming to literature, and because it is considered a benign substance, most experiments were carried out with calcium carbonate. Cobalt carbonate and copper carbonate were used to proof that the method also works for other multivalent ions (See the Supplementary Information). Particular focus of the study is on the importance of the buffering properties of the acid, and the propensity of its conjugated base to form complexes with the selected cations. ${ }^{32}$ Hydrochloric and nitric acid were selected as strong inorganic acids, with minimal buffering capacity. Acetic acid, citric acid, and lactic acid were selected as weaker acids, with larger buffering capacities. It should be noted that these acids can be obtained from sustainable sources. ${ }^{33-35}$ Glucono-delta-lactone (GDL) was selected as a gluconic acid precursor, ${ }^{36}$ because the extensive buffering properties in the $\mathrm{CaCO}_{3}-\mathrm{GDL}$ system is proven to result in a time-delayed release of calcium ions that produces more homogenous alginate gels. ${ }^{37}$ 
In this paper, we show that the bio-ionic crosslinking, induced by the carbonate-acid reaction, allows for facile and solvent/organic polymer-free fabrication of inorganic porous hollow fiber membranes. The fibers have very thin walls, superior mechanical strengths, and more narrow distributions of smaller pores, as compared to fibers obtained by previous methods.

\section{Experimental}

\section{Chemicals}

Alginic acid sodium salt from brown algae with medium viscosity, calcium carbonate, cobalt carbonate, copper carbonate (all Sigma-Aldrich); AKP-30 and AKP-50 $\alpha$-alumina powder with a mean particle size of $0.3 \mu \mathrm{m}$ and $0.1 \mu \mathrm{m}$ respectively (Sumitomo Chemicals); and de-ionized water (>18.2 $\mathrm{M} \Omega \mathrm{cm}$, Milli-Q Advantage A10, Millipore) were used to prepare spinning mixtures. Acetic acid (Merck), citric acid, glucono delta-lactone (GDL), hydrochloric acid, lactic acid, and nitric acid (All from Sigma-Aldrich) were used as proton source.

\section{Preparation of spinning mixture}

$0.15 \mathrm{~g}$ calcium carbonate was added to $82.5 \mathrm{~g}$ de-ionized water in a two-neck flask followed by stirring for 10 minutes. $12.5 \mathrm{~g}$ AKP-30 $\alpha$-alumina was added under continuous stirring and ultrasonic treatment, followed by stirring and ultrasonic treatment for 20 minutes (Branson 1800, $10 \mathrm{~min}, 40 \mathrm{kHz}$ ). 5.0 g sodium alginate was added in three steps to prevent the formation of alginate lumps. The mixture was stirred overnight to obtain a homogenous spinning mixture.

\section{Preparation of fibers}

Non-hollow alumina fibers were prepared by extruding the spinning mixture into solutions of acetic acid, citric acid, GDL, or lactic acid, with a pH of 1.7, 2, 3 and 4. For hydrochloric and nitric acid, a pH of $0.6,1,1.3$ and 2 was used. Upon contact with the acid gelation bath, crosslinking of the alginate occurs. After extrusion, samples were left overnight in the acid gelation bath. The cross-linked fibers were dried at room temperature for $24 \mathrm{~h}$ and were subsequently 
thermally treated in a STF16/610 tubular furnace (Carbolite) under an airflow of $100 \mathrm{~mL} \mathrm{~min}{ }^{-1}$. Using a heating rate of $5 \mathrm{C} \mathrm{min}^{-1}$, the fibers were dried at $110{ }^{\circ} \mathrm{C}$ for $60 \mathrm{~min}$, followed by further sintering at $1400{ }^{\circ} \mathrm{C}$ for 120 minutes.

Hollow alumina fibers were prepared through a similar procedure by spinning into a lactic acid solution with a $\mathrm{pH}$ of 1.7.The spinning mixture was pressurized in a stainless steel vessel, degassed for 30 minutes and left overnight. Spinning was carried out using a spinneret with an outer/inner diameter of 2.0/0.8 mm, using 2 bar nitrogen pressure and at ambient temperature. The air gap was $15 \mathrm{~mm}$ and the bore liquid flow rate was $14 \mathrm{~mL} \mathrm{~min}{ }^{-1}$. The bore liquid was taken from gelation bath, which consists of lactic acid with a $\mathrm{pH}$ of 1.7. After spinning, the fibers were left in the gelation bath overnight, dried and thermally treated following the procedure described above. In addition, fibers were prepared using NIPS ${ }^{14}$ and external gelation $^{17}$ as described elsewhere.

\section{SEM}

Scanning electron micrographs of the cross section morphology and the wall thickness of green and sintered fibers were obtained with a Zeiss Merlin FE-SEM or JEOL-JSM6010 Scanning Electron Microscope. To obtain a flat cross-section, the green compacts were soaked in ethanol and submersed in liquid nitrogen before fracturing. No further pre-treatment was done on the samples.

\section{TGA/DTA-MS}

Thermogravimetric analysis combined with differential scanning calorimetry analysis (TGA/DSC) was performed on a STA 449 F3 Jupiter ${ }^{\circledR}$ (Netzch) fitted with a TGA-DSC sample holder. Measurements were performed under a flow of $70 \mathrm{~mL} \mathrm{~min}{ }^{-1}$ synthetic air at a heating rate of $20^{\circ} \mathrm{C} \mathrm{min}^{-1}$ from room temperature to $1000{ }^{\circ} \mathrm{C}$. A temperature correction and sensitivity 
analysis using melting standards and a blank correction with an empty cup were carried out prior to the measurements. Small fragments of fibers that had been dried overnight under vacuum at $50{ }^{\circ} \mathrm{C}$ were used as sample and their mass was determined externally.

\section{Mechanical Strength}

The mechanical strength of the prepared hollow fibers was measured on a 5564A mechanical testing bench (Instron). Sintered fibers were picked randomly and broken to a length of approximately $5 \mathrm{~cm}$, followed by tested using a 4 point fixture, with an outer span $\left(L_{0}\right)$ of 20 $\mathrm{mm}$ and an inner span $\left(L_{\mathrm{i}}\right)$ of $10 \mathrm{~mm}$. The load was measured at constant extension rate $(2 \mathrm{~mm}$ $\min ^{-1}$ ) and the inner and outer diameters of the fiber were measured after fracture, using a digital caliper (Mitutoyo CD-15CPX, $\Delta d \pm 0.02 \mathrm{~mm}$ ). A sample set size of 30 samples was used.

The maximum bending strength is computed using Equation 1 , where $F_{j}$ is the force at fracture for specimen $j, L_{0}$ the distance between the two outer rollers, $K$ is defined by half the distance between the inner and outer span $K=\left(L_{o}-L_{i}\right) / 2$, and $d_{0, j}$ and $d_{\mathrm{i}, j}$ the outer and inner diameter of specimen $j$ :

$$
\sigma_{f, j}=\frac{16 F_{j} K d_{o, j}}{\pi\left(d_{o, j}^{4}-d_{i, j}^{4}\right)}
$$

\section{Nitrogen gas permeation}

Single-gas permeation was carried out using nitrogen (Linde Gas, Purity 4.0). Fibers were potted into 1/4" stainless steel tubing and sealed with glue (Epoxy, Araldite 2014) before they were mounted into a stainless steel module. The inside-out nitrogen flow was measured using a soap-film flow meter while the feed pressure was regulated using a pressure-reducing valve (Genie, 0-10 bar). The permeate side is assumed to be at atmospheric pressure. 


\section{Mercury intrusion}

The volume of mercury intruded was measured as function of the pressure using a Poremaster

PM-33-14 (Quantachrome®). The pore diameter corresponding to a certain pressure is calculated using Washburn’s equation from the measured volume mercury intruded $V_{\mathrm{Hg}}$ versus pressure $P$ using Matlab (Mathworks, R2014b) . The volume based pore size distribution is calculated via equation $2 .{ }^{38}$

$$
D_{v}(r)=\frac{\mathrm{d} V_{\mathrm{Hg}}}{\mathrm{d} P}\left|\frac{P^{2}}{2 \gamma \cos \theta}\right|
$$




\section{Results and discussion}

\section{Fiber consolidation}

Figure 2 shows the non-hollow fibers that result from injecting a calcium carbonate/alginate/alumina solution into the different gelation baths. The characteristics of the gels that are formed, and extent to which the inorganic particles are incorporated, show a strong dependency on the $\mathrm{pH}$ of the gelation bath and the acid strength. A maximum critical $\mathrm{pH}$ appears to exist to induce effective gelation. Below this $\mathrm{pH}$ a sufficient amount of calcium is released to form a stable and insoluble network. It should be noted that these critical pH values are below the $\mathrm{p} K_{\mathrm{a}}$ values of the sodium alginate building blocks, ( $(-\mathrm{D})$-mannuronic acid (3.38) and ( $\alpha$-L)-guluronic (3.65). This implies that the sodium alginate will be partly converted into alginic acid, ${ }^{39,40}$ which is known to form gels that are stabilized by intermolecular hydrogen bonds. This could further aid the cross-linking of the fibers. ${ }^{41}$

In the strong acids $\left(\mathrm{HCl}\right.$ and $\left.\mathrm{HNO}_{3}\right)$ rigid shapes are obtained at $\mathrm{pH}$ values below 1.3 (See the Supplementary Information, Figure S-3). At higher pH values, insufficient protons are available to liberate calcium ions, inhibiting the cross-linking of the fiber. For the strong acids the $\mathrm{pH}$ increases substantially when the sodium alginate mixture is injected in the acid solution. For the weak acids, a pH of 1.7 is already sufficient to obtain a rigid shape. The buffering properties of the weak acids allow for a continuing release of protons and subsequent calcium release. For the weak acids, the $\mathrm{pH}$ does not show a substantial increase when the sodium alginate mixture is injected in the acid solution. $\mathrm{pH}$ values in excess of 2 result in only partially cross-linked alginate gels. This lesser extent of crosslinking becomes more pronounced at higher $\mathrm{pH}$ values, until virtually no cross-linking is observed for fibers spun into acids with a $\mathrm{pH}$ of 4 . At a pH of 3, rigid structures are obtained for the acetic and lactic acid, whereas the structures for gluconic and citric acid result in a loosely packed gel that lacks mechanical integrity. Using citric and 
gluconic acid there will be competition between calcium uptake by the alginate and complexation of calcium ions by the conjugated base. ${ }^{23,42}$

Fibers taken from an acid bath, with a $\mathrm{pH}$ below the critical value, are flexible and have sufficient mechanical strength for handling. The microstructure of the fibers reveals a dense matrix for the citric and gluconic acid, whereas a more open structure is observed for acetic and lactic acid (See Figure S-6 in the Supplementary Information for the scanning electron micrographs.) Upon drying, the fibers’ diameter and flexibility reduced largely.

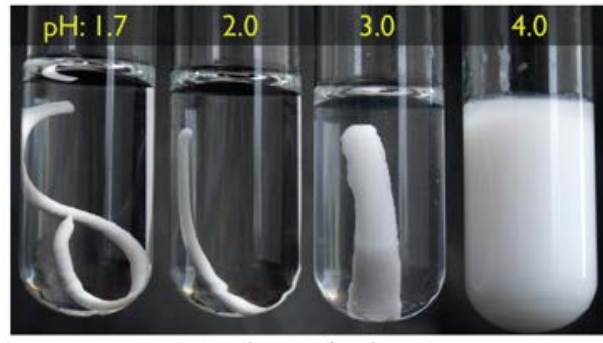

Lactic Acid

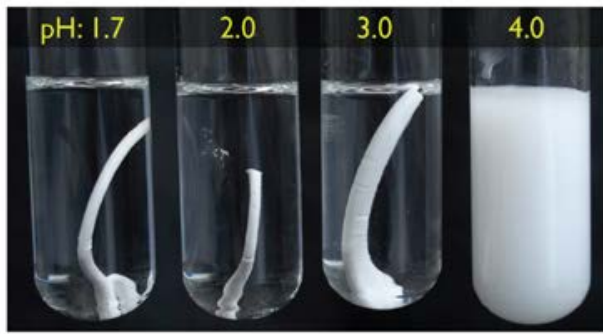

Acetic Acid

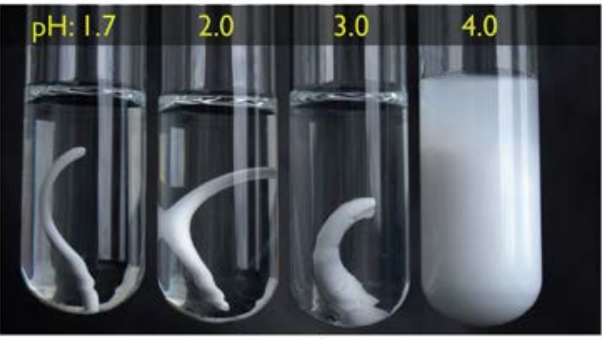

Citric Acid

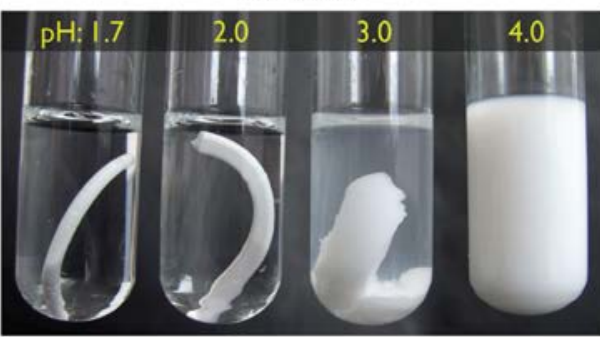

Gluconic acid

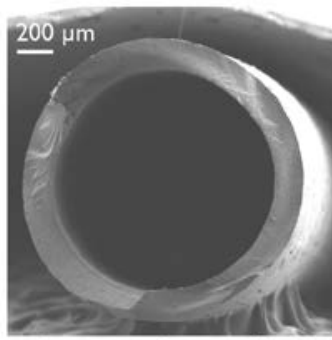

Lactic acid: green fiber

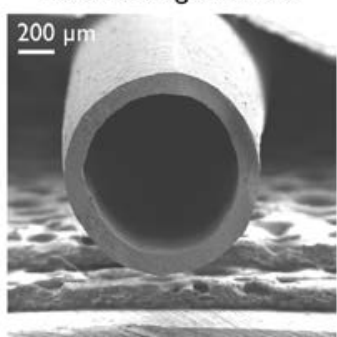

Lactic acid: sintered fiber

Figure 2. Left and middle panels: Photos of non-hollow fibers obtained from injecting a calcium carbonate/alginate/alumina solution into the different acids at different pHs; Right panels: Scanning Electron Micrographs of the green and sintered hollow fiber. Note: the contrast and brightness of the pictures has been adjusted to improve the visualization.

\section{Thermal treatment}

All fibers have been subjected to identical thermal treatment. For the fibers obtained from $\mathrm{HCl}$, $\mathrm{HNO}_{3}$, acetic acid or lactic acid and below the critical $\mathrm{pH}$, the thermal treatment resulted in well-shaped and structurally intact fibers. The fibers obtained from citric acid or GDL solutions, on the other hand, disintegrated into powder during the thermal treatment. Figure 3 shows the results of the TGA/DSC analysis of the dried fibers and powders (TGA/DSC data for $\mathrm{HCl}$ and $\mathrm{HNO}_{3}$ are not shown here but are given in the Supplementary Information, Figure S-4). Up to 
$150{ }^{\circ} \mathrm{C}$, the mass loss is below $1 \%$, and can be attributed to the last stages of drying of the fibers. Around $150{ }^{\circ} \mathrm{C}$, the onset of mass loss is found for all fibers, followed by a multi-step, exothermic mass loss. During these steps, the mass loss is significantly higher for citric acid and gluconic acid, than for the other acids.

For all experiments, the spinning mixture consisted of $28.4 \mathrm{wt} \%$ alginate, $70.8 \mathrm{wt} \%$ alumina, and $0.8 \mathrm{wt} \% \mathrm{CaCO}_{3}$ (on dry basis). For fully dried fibers, a maximum theoretical mass loss of 28.7\% would therefore be expected based on the spinning mixture, assuming that the calcium remains in the fibers in the form of $\mathrm{CaO}$. Only for $\mathrm{HCl}$ (23.61 wt\%), $\mathrm{HNO}_{3}$ (23.40 wt\%) and acetic acid (25.5 wt\%), the experimental mass loss is lower than the theoretical expectation. For all other acids, the mass losses are higher: $43.6 \mathrm{wt} \%$ for lactic acid, $62.8 \mathrm{wt} \%$ for citric acid and $64.4 \mathrm{wt} \%$ for gluconic acid.

Co-diffusion of the conjugated base to balance the charge of the diffusing protons does not explain the mass difference between theory and experiment, as this would result in a surplus mass loss of $0.4 \%$ (acetic acid), $0.7 \%$ (lactic acid), and 1.9\% (gluconic and citric acid). The adsorption of the acids onto the surface of alumina particles can also explain only a small amount of extra mass, because of the low surface area of the particles. ${ }^{43}$ Complexation of $\mathrm{Ca}^{2+}$ or $\mathrm{Na}^{+}$ions, ${ }^{32}$ would result in only low extra mass for lactic acid and acetic acid, but potentially results in much higher masses for the citric acid and GDL ions. In addition, the complexes formed would be able to hold a significant amount of water that may be released at higher temperatures than normally can be expected. ${ }^{44}$ Indeed, SEM micrographs of the green citric acid and GDL fibers (given in Figure S-6 in the Supplementary Information) suggest the presence of more non-alumina material. The higher water content that these layers may hold, could rationalize the surplus mass loss. ${ }^{26,45,46}$ At $250{ }^{\circ} \mathrm{C}$, the alginate starts to decompose, $, 4,48$ inducing a further release of the water as seen in the mass spectrum of the gasses evolving the sample (See the Supplementary Information, Figure S-5). Indeed, the recorded heat flow is 
comparatively low, which could be the result of the endothermic contribution of water evaporation.
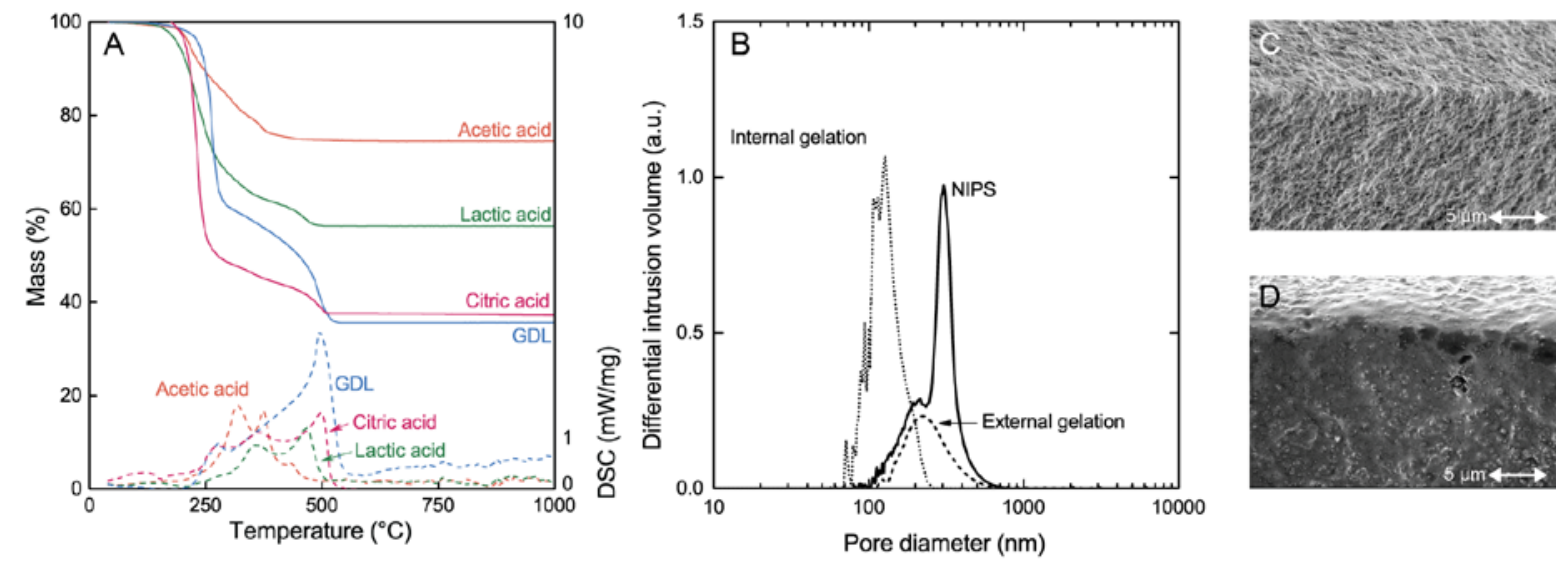

Figure 3. Thermal processing and structure of sintered fibers. A: TGA (solid lines) and DSC (dashed lines) curves of the cross-linked fibers obtained by spinning into the acid solution with $\mathrm{pH}$ of 1.7; B: Pore size of the sintered fiber spun into lactic acid (internal gelation), compared to standard spinning into a $\mathrm{CaCl}_{2}$ solution (external gelation), and a fiber obtained from nonsolvent-induced phase separation; C and D: Scanning electron micrograph of a green fiber prepared with lactic acid (C) and citric acid (D) at pH 1.7.

Figure 3B shows the pore size distribution of fibers prepared via internal gelation, external gelation, and NIPS. The data clearly show that the fiber prepared using internal gelation has smaller pore size as compared to fibers prepared using external gelation or NIPS. Pores in the range of 100-200 $\mathrm{nm}$ are observed, which agrees well with the original powder size of approximately $300 \mathrm{~nm}$ and the assumption of dense sphere packing. For fibers prepared using NIPS, it is known that the stacking of the particles is less dense, resulting in larger pore sizes using equivalent starting materials. ${ }^{14}$ The porosities of the internal, external and NIPS fibers are 29, 16 and 54\% respectively. (See the Supplementary Information, Table S-6)

The combination of spatially uniform and slow gel formation will aid the formation of a homogeneous and dense packed ceramic body. In addition, at the low $\mathrm{pH}$ values the alumina particles have a substantial positive surface charge. It is know that slow settling of charged alumina particles from a suspension allows for a obtaining well-defined homogeneous porous 
structures, with a narrow distribution of small pores ${ }^{49}$ and may prevent the formation of agglomerates by electrostatic stabilization. ${ }^{50}$

\section{Performance}

The results for the consolidation and the thermal treatment of the non-hollow fibers indicate that acetic acid and lactic acid are the best choices for the acid bath. These acids have sufficient buffering capacity, are available from sustainable sources, and generate fibers that show limited mass loss during thermal treatment. Given the pungent odor of acetic acid, preference is given to the use of lactic acid.

Thin $(110 \mu \mathrm{m})$, flexible, and defect free hollow fibers were obtained by pressing a $\mathrm{CaCO}_{3} /$ alginate/alumina solution through a spinneret into a lactic acid bath with a $\mathrm{pH}$ of 1.7 . The scanning electron micrographs in Figure S-5 of the Supplementary Information show that homogenous ceramic bodies are obtained that are virtually free from any large defects that might negatively affect mechanical strength.

Figure 4A shows the 4-point bending strengths for 30 of these hollow fibers after sintering. Based on the measured strength values, an empirical cumulative distribution is computed using Kaplan-Meijer estimates. ${ }^{51}$ A lot of scatter is present in the mechanical strength data. This scatter does not only originate from the defect distribution, but is presumably also a result of lateral variations in the dimensions and the structure of the fiber (See Supplementary Data, Figure S-8). Furthermore, two failure populations can be distinguished, suggesting different failure mechanisms. ${ }^{52}$ The presence of different failure populations might indicate distinctive microstructural features of the fibers, for instance the presence of small agglomerates. ${ }^{53}$ The presence of such small agglomerates is not apparent from the scanning electron micrographs.

By fitting the strength data with a normal distribution an average strength of $232.7 \pm 28.5$ (95\% CI) MPa is calculated, with a standard deviation of $77.6 \pm 15.6$ (95\% CI) MPa. These 
mechanical strength values are exceptionally high for $\mathrm{Al}_{2} \mathrm{O}_{3}$ hollow fibers. Typical values reported in literature range from $100-150 \mathrm{MPa},{ }^{1,54-56}$ and are generally obtained using a 3-point bending test. A direct comparison between strength numbers is in general not straightforward, because 3-point bending tests systematically result in higher strength values in comparison with 4-point bending tests. ${ }^{57}$ This further supports the high mechanical strength of the prepared fibers, which is a direct result of the elimination of the macro-voids that are inherently present in fibers prepared using NIPS, and the controlled homogenous stacking of the alumina particles obtained using internal gelation.

Figure 4B shows the normalized inside-out nitrogen permeance versus the arithmetic mean pressure inside the fiber wall. ${ }^{14}$ Some fiber-to-fiber differences are observed, likely due to slight variations in the wall thickness and diameter over the fiber' length. The estimated pore size $d_{p}$ and porosity-tortuosity ratio $\varepsilon / \tau$ are in between $179-207 \mathrm{~nm}$ and $0.04-0.09$, respectively (assuming cylindrical pores with unimodal pore size distribution, see the Supplementary Information, Table S-4). These obtained flow-average pore sizes are in excellent agreement with the $100-200 \mathrm{~nm}$ pore diameter values that were determined by mercury intrusion. The $\varepsilon / \tau$ values are slightly lower than those typically obtained in our labs for high quality alumina supports obtained by colloidal filtration of peptized alumina particles, which are $\sim 0.1$. For hollow fibers that are prepared by NIPS, much lower $\varepsilon / \tau$ values have been reported. These low values have been rationalized by the less homogeneous microstructure of these fibers, further undermining the assumption of a unimodal pore size distribution in these fibers. ${ }^{14}$ Under the assumption of a tortuosity of $3,{ }^{58-60}$ the porosity of the fibers obtained by internal gelation is calculated to be in the range $13-28 \%$, which agrees well with the value of $28.8 \%$ obtained using mercury intrusion (See the Supplementary Information Table S-6). 

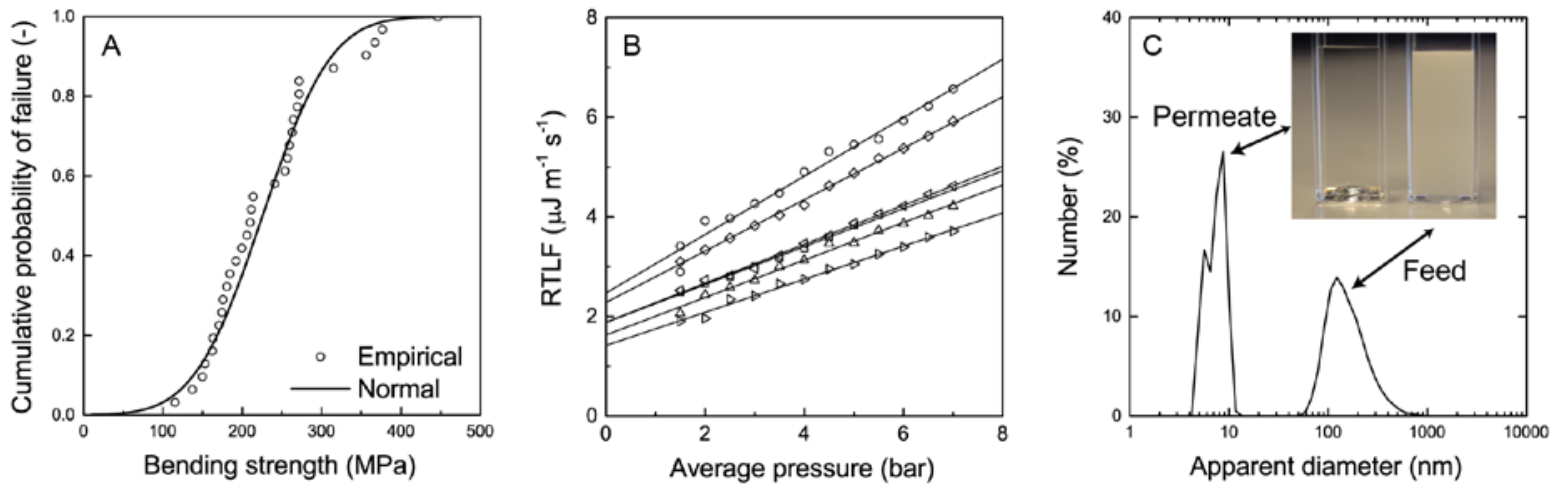

Figure 4. Panel A shows the observed mechanical strength of sintered hollow fibers (Lactic Acid, $\mathrm{pH}=1.7$, sintered in air for $1400^{\circ} \mathrm{C}, 2 \mathrm{~h}$ ) ranked to their probability of failure. The 30 different fibers are presented as spheres, whereas the solid line in A represents a normal distribution fitted to this data. Panel B shows the nitrogen permeation for 6 different fibers as function of the average pressure in the fiber wall $\left(P_{\mathrm{m}}=\left(P_{\text {feed }}+P_{\text {permeate }}\right) / 2\right)$. Panel C shows the feed (right vial) and permeate (left vial) of a separation experiment with an aqueous feed containing AKP-50 particles. The curve shows the apparent particle diameter of the particles in the feed and permeate.

The hollow fibers have various potential applications, including their use as catalyst or membrane support, and their direct use as a microfiltration membrane. The potential of these fibers as a microfiltration membrane is demonstrated by the filtration of an aqueous dispersion of AKP-50 alumina particles with a mean particle size of $100 \mathrm{~nm}$, in dead-end mode. Figure 4C shows the feed and permeate dispersions. The apparent difference in the turbidity of the solutions confirms that the particles are retained by the membrane. The particle size distribution of the feed and permeate shows that the peak is shifted significantly towards smaller particle sizes. The fact that only particles smaller than $10 \mathrm{~nm}$ are detected in the permeate shows the ability of the membrane to retain the particles with larger sizes. The size of the particles that can be separated is a direct result of the pore size of the fibers, which can be tuned by either adjusting the particle size of the inorganic starting material or by adjusting the thermal treatment program of the fiber. 


\section{Conclusion}

We developed a sustainable route for the fabrication of high performance inorganic porous hollow fibers without the use of organic solvents and harmful additives. The route is based on pressing an aqueous mixture of a carbonate, a sodium alginate, and inorganic particles through a spinneret into an acid bath. The low $\mathrm{pH}$ triggers the dissociation of the carbonate into multivalent cations and carbon dioxide. The multivalent cations are responsible for crosslinking of the alginate, which in turn arrests the inorganic particles. During a subsequent thermal treatment, the polymer is removed and the particles are sintered together

The acid in the coagulation bath is crucial for successful fiber preparation; not only is a sufficiently low $\mathrm{pH}$ of the acid bath required, it is also critical to select an acid with appropriate buffering capacity, and a conjugated base that has limited propensity to complexation with cations in order to fabricate high quality fibers.

By using this method, we are able to prepare alumina hollow fibers with outstanding properties, such as high mechanical strength, homogeneous morphology, and sharp distribution of small pores. These fibers have an extensive application landscape; they can for instance be used directly as a microfiltration membrane, or can be used as catalyst of membrane support material. Our new method substantially reduces the environmental impact of the fiber production process, because it only requires benign chemicals such as sodium alginate, lactic acid, and calcium carbonate that can be obtained from sustainable sources. 


\section{Acknowledgements}

This research is supported by the Dutch Technology Foundation STW, which is part of the

Netherlands Organization for Scientific Research (NWO), and which is partly funded by the Ministry of Economic Affairs.

\section{Supporting information}

Supporting information includes:

- Information about the fibers prepared by using alternative carbonates

- $\quad$ TGA/DSC data and Scanning Electron Micrographs of the prepared bio-polymers

- Details about the mechanical strength and gas permeation measurements.

This material is available free of charge via the Internet at http://pubs.acs.org. 


\section{References}

(1) Zhang, X.; Wang, D. K.; Lopez, D. R. S.; Diniz da Costa, J. C. Fabrication of nanostructured $\mathrm{TiO} 2$ hollow fiber photocatalytic membrane and application for wastewater treatment. Chem. Eng. J. 2014, 236, 314-322.

(2) Zhao, T.; Liu, Z.; Nakata, K.; Nishimoto, S.; Murakami, T.; Zhao, Y.; Jiang, L.; Fujishima, A. Multichannel TiO2 hollow fibers with enhanced photocatalytic activity. J. Mater. Chem. 2010, 20, 5095.

(3) Wung, N.; Acott, S. M.; Tosh, D.; Ellis, M. J. Hollow fibre membrane bioreactors for tissue engineering applications. Biotechnol. Lett. 2014, 2357-2366.

(4) Katuri, K. P.; Werner, C. M.; Jimenez-sandoval, R. J.; Chen, W.; Jeon, S.; Logan, B. E.; Lai, Z.; Amy, G. L.; Saikaly, P. E. A Novel Anaerobic Electrochemical Membrane Bioreactor (AnEMBR) with Conductive Hollow-fiber Membrane for Treatment of Low-Organic Strength Solutions. Environ. Sci. Technol. 2014, 48, 12833-12841.

(5) Li, J. L.; Chen, B. H. Review of CO2 absorption using chemical solvents in hollow fiber membrane contactors. Sep. Purif. Technol. 2005, 41, 109-122.

(6) Dindore, V. Y.; Brilman, D. W. F.; Versteeg, G. F. Hollow fiber membrane contactor as a gas-liquid model contactor. Chem. Eng. Sci. 2005, 60, 467-479.

(7) Kreulen, H.; Smolders, C. A.; Versteeg, G. F.; van Swaaij, W. P. M. Microporous hollow fibre membrane modules as gas-liquid contactors. Part 1. Physical mass transfer processes. J. Memb. Sci. 1993, 78, 197-216.

(8) Dutczak, S. M.; Tanardi, C. R.; Kopeć, K. K.; Wessling, M.; Stamatialis, D.

"Chemistry in a spinneret" to fabricate hollow fibers for organic solvent filtration. Sep. Purif. Technol. 2012, 86, 183-189.

(9) David, O.; Gendel, Y.; Wessling, M. Tubular macro-porous titanium membranes. J. Memb. Sci. 2014, 461, 139-145.

(10) Michielsen, B.; Chen, H.; Jacobs, M.; Middelkoop, V.; Mullens, S.; Thijs, I.; Buekenhoudt, a.; Snijkers, F. Preparation of porous stainless steel hollow fibers by robotic fiber deposition. J. Memb. Sci. 2013, 437, 17-24.

(11) Julbe, A.; Farrusseng, D.; Guizard, C. Porous ceramic membranes for catalytic reactors - Overview and new ideas. J. Memb. Sci. 2001, 181, 3-20.

(12) Wang, H.; Feldhoff, A.; Caro, J.; Schiestel, T.; Werth, S. Oxygen selective ceramic hollow fiber membranes for partial oxidation of methane. AIChE J. 2009, 55, 26572664.

(13) Buysse, C.; Kovalevsky, a.; Snijkers, F.; Buekenhoudt, a.; Mullens, S.; Luyten, J.; Kretzschmar, J.; Lenaerts, S. Development, performance and stability of sulfur-free, 
macrovoid-free BSCF capillaries for high temperature oxygen separation from air. $J$. Memb. Sci. 2011, 372, 239-248.

(14) Luiten-olieman, M. W. J.; Raaijmakers, M. J. T.; Winnubst, L.; Bor, T. C.; Wessling, M.; Nijmeijer, A.; Benes, N. E. Towards a generic method for inorganic porous hollow fibers preparation with shrinkage-controlled small radial dimensions, applied to Al2O3, Ni, SiC, stainless steel, and YSZ. J. Memb. Sci. 2012, 407-408, 155-163.

(15) Husain, S.; Koros, W. J. Macrovoids in Hybrid Organic/Inorganic Hollow Fiber Membranes. Ind. Eng. Chem. Res. 2009, 48, 2372-2379.

(16) Tan, X.; Liu, S.; Li, K. Preparation and characterization of inorganic hollow fiber membranes. J. Memb. Sci. 2001, 188, 87-95.

(17) Shukla, S.; de Wit, P.; Luiten-Olieman, M. W. J.; Kappert, E. J.; Nijmeijer, A.; Benes, N. E. Synthesis of Porous Inorganic Hollow Fibers without Harmful Solvents. ChemSusChem 2015, 8, 251-254.

(18) Grant, G. T.; Morris, E. R.; Rees, D. A.; Smith, P. J. C.; Thom, D. Biological interactions between polysaccharides and divalent cations: The egg-box model. FEBS Lett. 1973, 32, 195-198.

(19) Skjåk-Bræk, G.; Grasdalen, H.; Smidsrød, O. Inhomogeneous polysaccharide ionic gels. Carbohydr. Polym. 1989, 10, 31-54.

(20) Draget, K. I.; Skjåk-Braek, G.; Smidsrød, O. Alginate based new materials. Int. J. Biol. Macromol. 1997, 21, 47-55.

(21) Vandenberg, G. W.; De La Noüe, J. Evaluation of protein release from chitosanalginate microcapsules produced using external or internal gelation. J. Microencapsul. 2001, 18, 433-441.

(22) Chan, L. W.; Lee, H. Y.; Heng, P. W. S. Production of alginate microspheres by internal gelation using an emulsification method. Int. J. Pharm. 2002, 242, 259-262.

(23) Paques, J. P.; Sagis, L. M. C.; van Rijn, C. J. M.; van der Linden, E. Nanospheres of alginate prepared through w/o emulsification and internal gelation with nanoparticles of CaCO3. Food Hydrocoll. 2014, 40, 182-188.

(24) Liu, X. D.; Yu, W. Y.; Zhang, Y.; Xue, W. M.; Yu, W. T.; Xiong, Y.; Ma, X. J.; Chen, Y.; Yuan, Q. Characterization of structure and diffusion behaviour of Ca-alginate beads prepared with external or internal calcium sources. J. Microencapsul. 2002, 19, 775782.

(25) Straccia, M. C.; d’Ayala, G. G.; Romano, I.; Laurienzo, P. Novel zinc alginate hydrogels prepared by internal setting method with intrinsic antibacterial activity. Carbohydr. Polym. 2015, 125, 103-112. 
(26) Lee, K. Y.; Rowley, J. A.; Eiselt, P.; Moy, E. M.; Bouhadir, K. H.; Mooney, D. J. Controlling mechanical and swelling properties of alginate hydrogels independently by cross-linker type and cross-linking density. Macromolecules 2000, 33, 4291-4294.

(27) Golmohamadi, M.; Wilkinson, K. J. Diffusion of ions in a calcium alginate hydrogelstructure is the primary factor controlling diffusion. Carbohydr. Polym. 2013, 94, 8287.

(28) Cathell, M. D.; Schauer, C. L. Structurally Colored Thin Films of Ca 2+-Cross-Linked Alginate. Biomacromolecules 2007, 8, 33-41.

(29) Padol, A. M.; Maurstad, G.; Draget, K. I.; Stokke, B. T. Delaying cluster growth of ionotropic induced alginate gelation by oligoguluronate. Carbohydr. Polym. 2015, 133, 126-134.

(30) Kilan, K.; Warszyński, P. Thickness and permeability of multilayers containing alginate cross-linked by calcium ions. Electrochim. Acta 2014, 144, 254-262.

(31) Choi, B. Y.; Park, H. J.; Hwang, S. J.; Park, J. B. Preparation of alginate beads for floating drug delivery system: Effects of CO2 gas-forming agents. Int. J. Pharm. 2002, 239, 81-91.

(32) Bazin, H.; Descotes, G.; Bouchu, A.; Petit-Ramel, M. Comparison of calcium complexation of some carboxylic acids derived from D glucose and D -fructose. Can. J. Chem. 1995, 73, 1338-1347.

(33) Lomate, S.; Katryniok, B.; Dumeignil, F.; Paul, S. High yield lactic acid selective oxidation into acetic acid over a Mo-V-Nb mixed oxide catalyst. Sustain. Chem. Process. 2015, 3, 5.

(34) Dhillon, G. S.; Brar, S. K.; Verma, M.; Tyagi, R. D. Utilization of different agroindustrial wastes for sustainable bioproduction of citric acid by Aspergillus niger. Biochem. Eng. J. 2011, 54, 83-92.

(35) Morales, M.; Dapsens, P. Y.; Giovinazzo, I.; Witte, J.; Mondelli, C.; Papadokonstantakis, S.; Hungerbuhler, K.; Perez-Ramirez, J. Environmental and economic assessment of lactic acid production from glycerol using cascade bio- and chemocatalysis. Energy Environ. Sci. 2015, 8, 558-567.

(36) Pocker, Y.; Green, E. Hydrolysis of d-glucono-d-lacone. J. Am. Chem. Sociecity 1972, 78, 113-119.

(37) Kuo, C. K.; Ma, P. X. Ionically crosslinked alginate hydrogels as scaffolds for tissue engineering: Part 1. Structure, gelation rate and mechanical properties. Biomaterials 2001, 22, 511-521.

(38) León y León, C. A. New perspectives in mercury porosimetry. Adv. Colloid Interface Sci. 1998. 
(39) Haug, A.; Myklestad, S.; Larsen, B.; Smidsrød, O.; Eriksson, G.; Blinc, R.; Paušak, S.; Ehrenberg, L.; Dumanović, J. Correlation between Chemical Structure and Physical Properties of Alginates. Acta Chem. Scand. 1967, 21, 768-778.

(40) Chan, L. W.; Lee, H. Y.; Heng, P. W. S. Mechanisms of external and internal gelation and their impact on the functions of alginate as a coat and delivery system. Carbohydr. Polym. 2006, 63, 176-187.

(41) Draget, K. I.; Skjåk-Bræk, G.; Stokke, B. T. Similarities and differences between alginic acid gels and ionically crosslinked alginate gels. Food Hydrocoll. 2006, 20, 170-175.

(42) Mansour, S. A. A. Thermal decomposition of calcium citrate tetrahydrate. Thermochim. Acta 1994, 233, 243-256.

(43) Hidber, P. C.; Graule, T. J.; Gauckler, L. J. Citric Acid - A dispersant for Aquous Alumina Suspensions. J. Am. Chem. Soc. 1996, 79, 1857-1867.

(44) Brosnan, D. A.; Robinson, G. C. Introduction to Drying of Ceramics; Wiley-American Ceramic Society, 2003.

(45) Lee, Y. K.; Mooney, D. J. Alginate : properties and biomedical applications. Prog Polym Sci. 2013, 37, 106-126.

(46) Bajpai, S. K.; Tankhiwale, R. Investigation of water uptake behavior and stability of calcium alginate/chitosan bi-polymeric beads: Part-1. React. Funct. Polym. 2006, 66, 645-658.

(47) Zohuriaan, M. J.; Shokrolahi, F. Thermal studies on natural and modified gums. Polym. Test. 2004, 23, 575-579.

(48) Said, A. A.; Hassan, R. M. Thermal decomposition of some divalent metal alginate gel compounds. Polym. Degrad. Stab. 1993, 39, 393-397.

(49) Benes, N. E. Mass transport in thin supported silica membranes; 2000.

(50) Hidber, P. C.; Graule, T. J.; Gauckler, L. J. Influence of the dispersant structure on properties of electrostatically stabilized aqueous alumina suspensions. Journal of the European Ceramic Society, 1997, 17, 239-249.

(51) Kaplan, E. L.; Meier, P. Nonparametric Estimation from Incomplete Observations. J. Am. Stat. Assoc. 1958, 53, 457-481.

(52) ASTM. C1239-13 Standard Practice for Reporting Uniaxial Strength Data and Estimating Weibull Distribution Parameters for Advanced Ceramics. ASTM International, West Conshohocken, PA, 2014, 1-18.

(53) Genet, M.; Houmard, M.; Eslava, S. A two-scale Weibull approach to the failure of porous ceramic structures made by robocasting: possibilities and limits. J. Eur. Ceram. Soc. 2013, 33, 679-688. 
(54) Jiansheng, L.; Lianjun, W.; Yanxia, H.; Xiaodong, L.; Xiuyun, S. Preparation and characterization of Al2O3 hollow fiber membranes. J. Memb. Sci. 2005, 256, 1-6.

(55) Liu, S.; Li, K.; Hughes, R. Preparation of porous aluminium oxide (Al2O3) hollow fibre membranes by a combined phase-inversion and sintering method. Ceram. Int. 2003, 29, 875-881.

(56) Fung, Y.-L. E.; Wang, H. Nickel aluminate spinel reinforced ceramic hollow fibre membrane. J. Memb. Sci. 2014, 450, 418-424.

(57) Rodrigues, S. A.; Ferracane, J. L.; Della Bona, Á. Flexural strength and Weibull analysis of a microhybrid and a nanofill composite evaluated by 3- and 4-point bending tests. Dent. Mater. 2008, 24, 426-431.

(58) Mota, M.; Teixeira, A.; Yelshin, A. Image analysis of packed beds of spherical particles of different sizes. Sep. Purif. Technol. 1999, 15, 59-68.

(59) Khirevich, S.; Höltzel, A.; Daneyko, A.; Seidel-Morgenstern, A.; Tallarek, U. Structure-transport correlation for the diffusive tortuosity of bulk, monodisperse, random sphere packings. J. Chromatogr. A 2011, 1218, 6489-6497.

(60) Bertei, A.; Nucci, B.; Nicolella, C. Effective transport properties in random packings of spheres and agglomerates. Chem. Eng. Trans. 2013, 32, 1531-1536. 


\section{For Table of Contents Use Only}

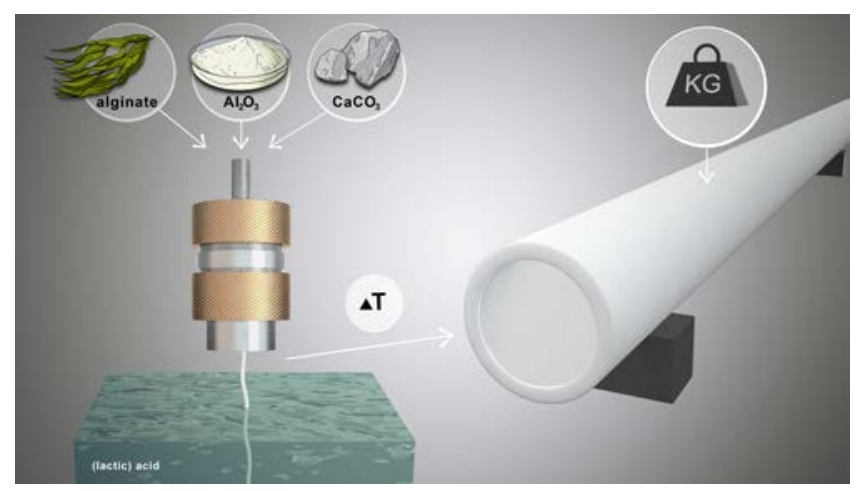

\section{A sustainable route to inorganic porous hollow fibers with superior properties}

Hussein Qasim Hussein, Patrick de Wit, Emiel J. Kappert, Nieck E. Benes

Fabrication of inimitable inorganic porous hollow fibers, by pH-triggered ionic cross-linking followed by thermal treatment, using sustainable feed materials. 